\title{
Prediction of long-term subgrade deformation under cyclic loadings with the implicit-explicit transition method using finite elements
}

\author{
K. Zhuang ${ }^{1}$, G. Quanmei ${ }^{1}$ \& W. Yang ${ }^{2}$ \\ ${ }^{1}$ Key Laboratory of Road and Traffic Engineering of the \\ Ministry of Education, TongJi University, China \\ ${ }^{2}$ China Urban Construction Design and Research Institute, \\ China
}

\begin{abstract}
Calculating accumulated soil deformation caused by millions of cyclic loadings with finite element simulating calculation is usually limited by storage space and physical error accumulation. The implicit method with which the constitutive relation of soil can be well described in fact cannot satisfy the finite element calculation on millions of times of cyclic loadings. With the research on the long-term deformation of soil under cyclic loadings, it is evident that this process has a similar character with the process of creep deformation of materials. In addition, the two experience expressions describing the two deformation process are also analogues. Based on these, a new idea to forecast soil deformation under high accumulated cyclic loadings is proposed in this paper. With this idea, the time-wasting and space-wasting problems in finite element calculation implementation is solved. Comparing the finite element calculation results and the dynamic triaxial experiment results, the accuracy of the idea is proved. With the comparison between the practical measured results of detection on highspeed railway section and finite element calculation results, the idea is further proved that it can represent the regularity and tendency of soil deformation under long-term cyclic loadings on a high-speed railway.
\end{abstract}

Keywords: high-speed railway, cyclic loadings, deformation prediction, finite element simulating calculation. 


\section{Introduction}

In the research on the high-speed railway, prediction of subgrade deformation usually carries great weight. During the deformation process, construction settlement is normally important but with the development of further research, the long-term accumulated deformation of soil caused by vehicle cyclic loadings as part of post-construction is more and more valued. Especially as the higher the speed of vehicles is developed, a tiny deformation may even develop into a great settlement. Therefore strict control of deformation is required to guarantee the comfortableness, safeness and stable operation in railway construction.

For the reason that it's hard to get the regularity and tendency of soil deformation with the help of long-term field test data solely, laboratory test is proposed to assist as the most accurate method in subgrade service life prediction research because of its real soil condition feedback. For example, Qian et al. [1] studied the railroad ballast vibration and deformation based on the months of field test and large scale triaxial test. It is long time and large resource cost but more reliable. With this method, the accuracy of the model can be guaranteed indeed and some empirical equations which getting from laboratory test result fitting are achieved. But soil sample for the laboratory test like dynamic tri-axial test can only support almost 10 thousand times cyclic loadings because of its scale. New method is eagerly needed to reflect the long-term dynamic influence with millions of cyclic loadings. With the development of finite element method and its application, finite element simulated calculation method is used in this field.

In the finite element simulated calculation, the process of soil deformation under cyclic loadings can be simulated. The cyclic loadings of vehicle are normally equalled by sine curve or triangular curve which is much easier to be exerted. And this equivalence has been proved reasonable by researchers [2-4]. And the key point in simulation and modeling is to make it possible to reflect the soil deformation under millions of cyclic loadings.

Normally, a soil constitutive model needs to be chosen as material in finite element calculation. Method with this model is named implicit method which is accords with the accuracy requirement of calculation. But most constitutive model within the finite element software is too simple to reflect the soil strain under tiny loadings. And to satisfy the soil characteristics in finite element simulation, complicated constitutive soil model need to be secondary developed. Even the constitutive model can describe the soil strain, millions of under cyclic loadings will produce large number of data which is space and time cost, not mention the physical system error accumulated during calculation.

In light of this, to ignore the single influence of each cyclic loading and focus on the tendency of plastic deformation of the process, in finite element simulating calculation the constitutive modal is replaced by pseudo static analysis method which normally expressed by empirical equations mentioned before. And this method accordingly called explicit method. Comparing with the implicit method the explicit method is much more convenience in calculation. 
Although once the specific value of each parameter is confirmed, the predicting result is believable. But the implicit method is still needed to reconfirm the strain-stress relationship during soil deformation. To assemble the benefits of the two methods, a better way which combines explicit method with implicit method is suggested by Wichtmann et al. [5]. With this method, stress and cyclic loading relationship will be achieved (as shown in Figure 1).

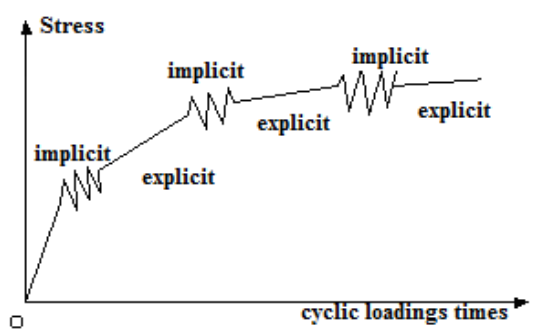

Figure 1: Curves of the relationship between stress and cyclic loadings times with the implicit-explicit method.

To realize the theoretical implicit-explicit method in finite element simulated calculation is another key point because usually there is no corresponding model in finite element software and secondary development is inevitable which bringing great difficulties to the calculation. In this paper, a convenience method without secondary development is proposed, and it can not only remain the accuracy of implicit method but also increase the calculation efficiency of longterm soil deformation under cyclic loadings.

\section{Proposal of explicit method in long-term soil deformation}

In the research of Wichtmann et al. [6], the long-term deformation under cyclic loadings could be divided into two parts: the vibrated part and the tendency part $[7,8]$. The former expresses the elastic deformation with certain amplitude, and the vibrated part actually can be ignored for long-term research. And the latter one expresses the plastic deformation which is the main part of long-term deformation. And the tendency part which described by relationship between loadings times and strain actually can be calculated and predicted by the empirical equations mentioned before.

Based on former research, the most typical empirical equation is the indexmodel proposed by Monismith and Long [9]. After that, through long time theoretical development and laboratory experiments, the following researchers $[10,11]$ have confirmed that dynamic deviatoric stress, static destroy deviatoric stress and so on are all connected with the soil deformation under cyclic loadings, and as influencing factors they have been expressed as parameters within the Monismith modifier empirical equation (1). It is considered well to predict the accumulated deformation and perfectly to represent the curve of dynamic triaxial experiment. 


$$
\varepsilon^{p}=a\left(\frac{q_{d}}{q_{f}}\right)^{m}\left(1+\frac{q_{s}}{q_{f}}\right)^{n} N^{b}
$$

In this equation, all the parameters come from experiments. $q_{s}$ is the static deviatoric stress, $q_{d}$ is the dynamic deviatoric stress, $q_{f}$ is the static failure deviatoric stress, a as a parameter effects the plastic strain; b as a parameter effects the plastic strain by controlling the increasing rate of loadings times $\mathrm{N} ; \mathrm{m}$ and $\mathrm{n}$ are index parameters by influencing dynamic and static deviatoric stress. According to the research of Chai and Miura [10], $n=1$ when the accumulated plastic strain is linear to the initial static deviatoric stress.

Although it has been proved that the Monismith modifier equation can well express the tendency of soil deformation under long-term dynamic load, there is no appropriate model to represent the equation directly with the explicit algorithm in most of finite element software unless complicated secondary development. So if there were an equivalent model which can instead of the explicit method to represent the tendency part of the soil plastic deformation, the simulating process will be greatly simplified. Therefore, the idea taking static implicit creep model instead of dynamic explicit soil model is proposed in finite element simulated calculation.

This replacement theoretically based on two common points. Firstly, creep deformation curve normally has similar characteristics with the soil deformation curve under long-term cyclic loadings, which means the empirical equations express the two processes even has the same form. And secondly, the creep model has to be comprehensively applied in most of the finite element simulation software, which provide a convenience to realize the replacement in finite element implementation.

Creep deformation has several different models. the most similar one with the soil long-term deformation is the "time hardening" creep which means the creep becomes harder and harder with the time accumulation. There is an equation to express this process as (2)

$$
\dot{\bar{\varepsilon}}_{c r}=c_{1} \sigma^{C_{2}} t^{C_{3}}
$$

In this equation, the rate of strain change is a function of time, and the equation can be integrated as (3):

$$
\bar{\varepsilon}_{c r}=\frac{c_{1}}{1+c_{3}} \sigma^{C_{2}} t^{C_{3}+1}
$$

Comparing with the equation (1), they all represent the unchangeable strain with the same equation form. In the equation (1), plastic strain is a function of loadings times $\mathrm{N}$ which effects with fixed-frequency. So the plastic strain is essentially also a function of time. So it's obviously that there is corresponding relationship between parameters of equation (1) and (3). And the relationships can be expressed by the following equations. 


$$
\left\{\begin{array}{c}
\frac{c_{1}}{1+c_{3}}=a\left(1+\frac{q_{s}}{q_{f}}\right) \text { when }(n=1) \\
c_{2}=m \\
c_{3}+1=b \\
N f=t
\end{array}\right.
$$

In equation (4), $f$ is the frequency of cyclic loadings. Add to that, normally the "temperature" will be considered in creep as a factor, but in soil long-term deformation under cyclic loadings it is an unnecessary factor. So the temperature factor in (3) is ignored.

If this replacement can be proved reasonable, it will provides a possibility to express the tendency of soil long-term deformation under cyclic loadings directly in finite element simulated calculation.

\section{Reasonability of the replacement testification and improvement}

First, dynamic tri-axial experiment is done in laboratory with typical clay borrowed from Ningbo. The results are fitted as Figure 2 and parameters of the equation (1) can be confirmed with the fitting method at a relatively high level.

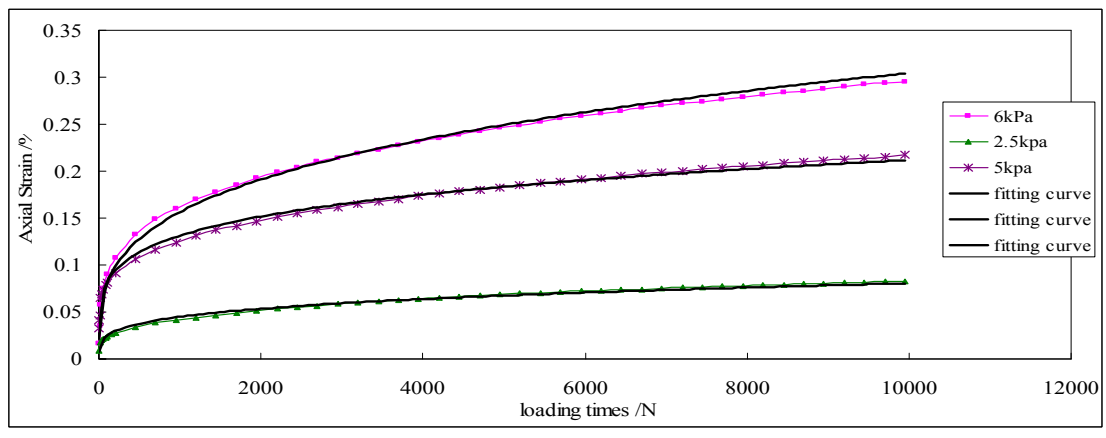

Figure 2: Fitting curves of dynamic triaxial experiment with different level of high-cyclic dynamic load.

Then to test the hypothesis, frequency-used finite element software ANSYS is taken as tool. Firstly a soil sample is modeled in ANSYS as the one in dynamic tri-axial experiment. Then the "time hardening creep" constitutive model is chosen in ANSYS as material.

In ANSYS, the "time hardening creep" is based on the Euler lead method, which means that the strain of next step is calculated by the strain and stress of the last time node. This procedure repeats in certain step length to circularly simulate the further process. 
Following the method above-mentioned, exchange all the parameters of (1) into (3) by the relationships (4). With "time hardening creep" constitutive model, simulate $\mathrm{N}=14000$ times cyclic loadings.

Comparing the simulated calculation result with real test result as shown in Figure 3, the strain magnitudes can be considered correspondence within limited cyclic loadings especially at the beginning of the process. There is also some deviation because of the difference between static and dynamic models. A dynamic coefficient will be introduced if necessary. With the test, to replace explicit soil deformation with creep constitutive model is feasible.

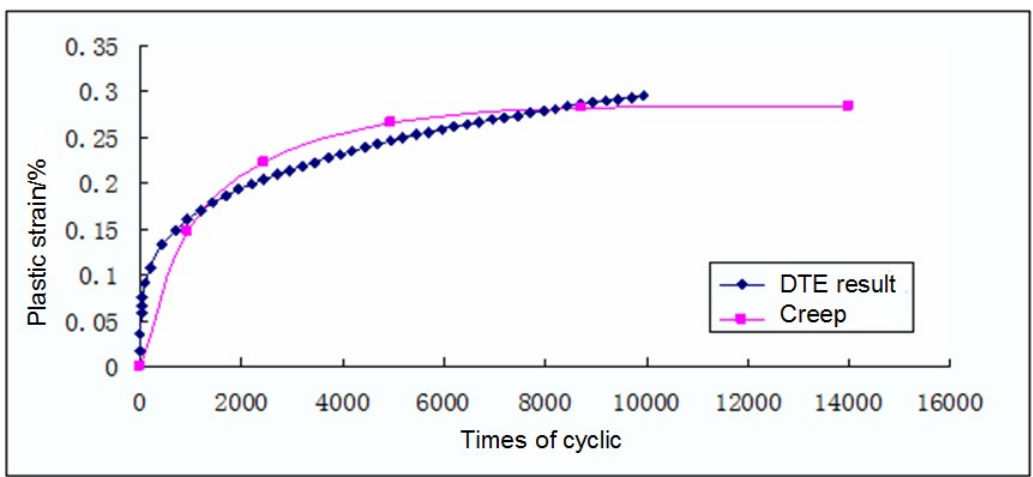

Figure 3: Results comparison between the real dynamic triaxial experiment and ANSYS.

With this replacement, the process of finite element simulation will be indeed convenience, but there is still a critical defect. The stress-strain changing relationships cannot be achieved during the simulation. But to adopt the changed stress field during the calculation is still necessary. An improvement method which switches between implicit and explicit methods is proposed.

In the process firstly the implicit method is applied with soil constitutive model, with this process the stress field could be confirmed. Then the explicit method is applied with creep constitutive model, with this process the tendency of soil deformation under cyclic loadings could be figured as Figure 4. At the

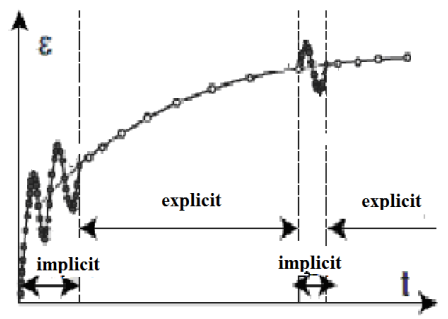

Figure 4: Equivalent curve of soil strain under cyclic loadings. 
switch point re-modeling method is taken to realize the transition. Repeating the above process, the equivalent curve of long-term soil deformation under cyclic loadings can be simulated in finite element implementation.

This method realizes the time-saving and data space-saving purpose meanwhile can predict credible soil deformation in ANSYS.

\section{Practical application in engineering with the improved method}

With this improved method, the subgrade long-term deformation of high-speed railway becomes predictable with finite element simulated calculation in practical engineering. In research of the Shanghai-Nanjing high-speed railway, which has have completed and opened to traffic in 2010, a section (DK20+900) of subgrade is picked (as shown in Figure 5). This section has been reinforced by the CFG piles with $0.5 \mathrm{~m}$ diameter piles and distributed as square shape. The height of subgrade is $2.7 \mathrm{~m}$ and the hardcore bed is $0.6 \mathrm{~m}$ with two levels bilateral geotextile. Soil prospect data and dynamic parameters of pile and soil are shown in Tables 1 and 2.

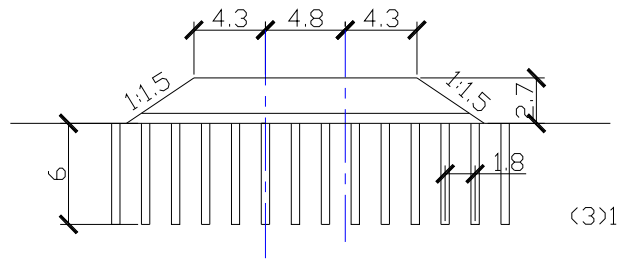

Figure 5: DK20+900 section of the Shanghai-Nanjing railway.

Table 1: $\quad$ Soil prospect data of section DK20+900.

\begin{tabular}{|c|c|c|}
\hline Id. & Name & Characteristics \\
\hline (3) 1 & a1Q3 silty clay & tawny, hard plastic, $\sigma_{0}=220 \mathrm{kPa}$, III level \\
\hline (4) 1 & $\begin{array}{c}\text { argillaceous } \\
\text { sandstone }\end{array}$ & taupe completely weathered, $\sigma_{0}=250 \mathrm{kPa}$, III level \\
\hline
\end{tabular}

Table 2: Dynamic parameter of pile and soil.

\begin{tabular}{|c|c|c|c|}
\hline & Dynamic elastic modulus/MPa & $\gamma / \mathrm{kN} / \mathrm{m}^{3}$ & Poison rate \\
\hline (3) silty clay & 30 & 20.2 & 0.3 \\
\hline piles & 40000 & 24 & 0.15 \\
\hline hardcore bed & 504 & 24 & 0.15 \\
\hline soil of embankment & 180 & 20 & 0.25 \\
\hline
\end{tabular}


To simulate the reinforcement area, modeling the single pile and soil around in the middle of the section and calculate with the principle of symmetry. Take the pile as end-bearing pile and Pick 5 intermediate nodes from soil between piles (as shown in Figure 6) as monitoring point to analyze the vertical displacement and plastic strain.

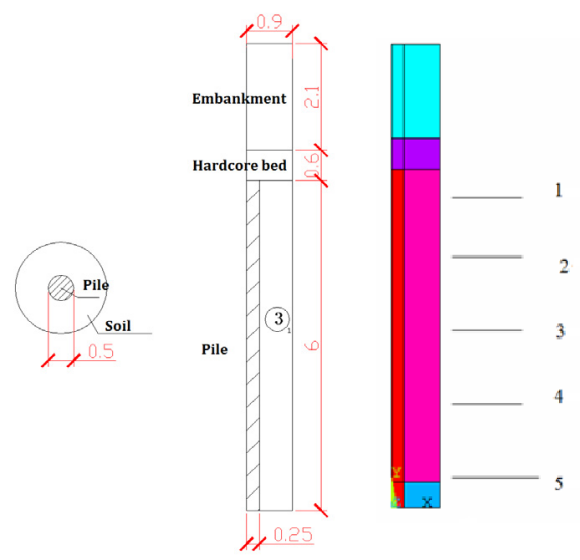

Figure 6: Modeling explanation and node-to-measure.

When cyclic loadings are provided in the reinforcement area, stress between soil and piles will be redistributed.

In equation (1), $\mathrm{N}$ accords to operating period, vehicle number, grouping number of each vehicle. And assumed that one wheelset causes one loading. In the Shanghai-Nanjing high-speed railway line section, vehicle grouping number is usually 16 with 32 wheelsets, there are 119 pairs vehicle pass through the section for one day. So the loadings number of each day could be calculated as: $\mathrm{N}=32 \times 238=7616$ times. Complete the simulation in ANSYS with this data and the subgrade accumulated deformation with loadings increasing is shown as Figure 7.

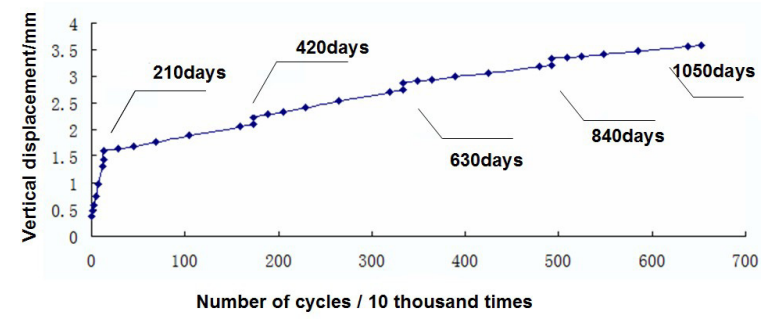

Figure 7: DK20+900 section vertical displacement increasing with number of cycles. 
Comparing this result with the measured deformation of this section after 396 days operation as Figure 8 shown, the calculated deformation of subgrade is $1.6 \mathrm{~mm}$ and the measured deformation is $1.4 \mathrm{~mm}$. The finite element calculation result could be considered acceptable and credible.

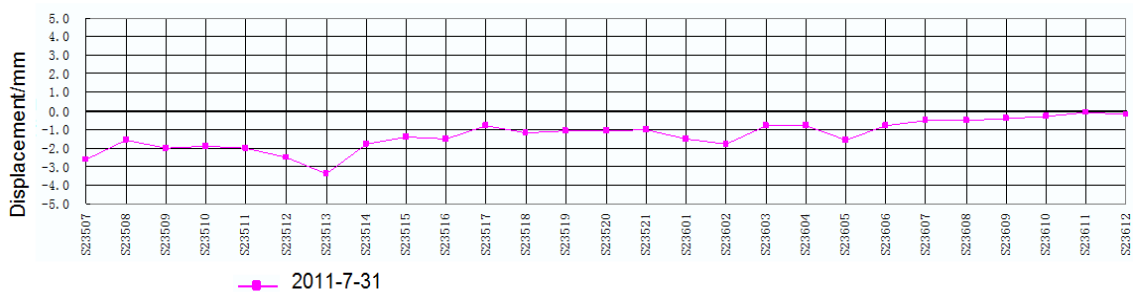

Figure 8: Settlement measured data of relevant sections of the ShanghaiNanjing railway.

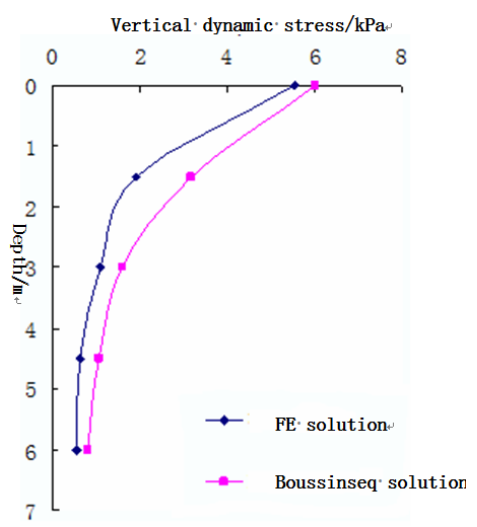

Figure 9: Vertical dynamic stress along depth of No.14 node calculated result.

Calculate the stress of soil between piles with both Boussinseq-Mindlin analytical method and ANSYS simulation, the result comparison is shown in Figure 9. The stress varies with the soil depth with the same tendency, which proved that this method is effective for pile-net stress calculation.

\section{Summary}

In conclusion, the implicit-explicit joint finite element simulate method is convenience and relatively accurate to predict the long-term deformation under cyclic loadings and stress distribution of railway subgrade. With this method, it's not only time-saving and data-space-saving within finite element calculation, but also proved can be used in practical engineering in the high-speed railway subgrade lifetime prediction. 


\section{Acknowledgements}

The authors are grateful for the financial support of Minister of Railway Science and Technology Research and Development Program, Grant, 2010G003-A, and the financial support of the Specialized Research Fund for the Doctoral Program of Higher Education, Grant, 20110072110026 is also appreciated.

\section{References}

[1] Yu Qian, Erol Tutumluer, Hai Huang, Ballast vibrations and deformations due to different train loading scenarios studied using the discrete element method. Proc. of 5th Int. Symp. on Environmental Vibration, eds. Zhai Wanming, Takemiya H, De Roeck G \& Tutumluer E, Elsevier: Science Press, pp. 613-619, 2011.

[2] Huang Mao-song, Li Jin-jun, Lu Xing-zhao. Cumulative deformation behaviour of soft clay in cyclic undrained tests. Chinese Journal of Geotechnical Engineering, 27(8), pp. 891-895, 2006.

[3] Tang Yiqun, Wang Yanling, Huang Yu, Dynamic strength and dynamic stress-strain relation of silt soil under traffic loading. Journal of Tongji University (Natural Science), 12(z1), pp. 99-101, 2004.

[4] Gong Quanmei, Liao Caifeng, Zhou Shunhua. Test of foundation soil dynamic pore water pressure under metro vehicle load. Chinese Journal of Rock Mechanics and Engineering in recent years, 20(z), pp. 1154-1157, 2001.

[5] T Wichtmann, A Niemunis, TH Triantafyllidis. Strain accumulation in sand due to cyclic loading: On the effect of monotonic and cyclic preloading. Soil Dynamics and Earthquake Engineering, 30(8), pp. 736$745,2010$.

[6] T Wichtmann, HA Rondon, A Niemunis, TH Triantafyllidis, A Lizcano. Prediction of Permanent Deformations in Pavements Using a High-Cycle Accumulation Model. Journal of Geotechnical and Geoenvironmental Engineering, 136(5), pp. 728-740, 2010.

[7] T Wichtmann, A Niemunis, TH Triantafyllidis. Strain accumulation in sand due to cyclic loading: drained triaxial tests. Soil Dynamics and Earthquake Engineering, 25(12), pp. 967-979, 2005.

[8] T Wichtmann, A Niemunis, TH Triantafyllidis. Strain accumulation in sand due to cyclic loading: Drained cyclic tests with triaxial extension. Soil Dynamics and Earthquake Engineering, 27(1), pp. 42-48, 2007.

[9] Monismith CL, Long F, Overlay design for cracked and seated Portland cement concrete (PCC) Pavement-interstate route 710, Technical Memorandum TM UCB PRC 99-3, Pavement Research Center, Institute for Transportation Studies, University of California, Berkeley 1999.

[10] Chai JC, Miura N, Traffic-load-induced Permanent Deformation of Road on Soft Soil, Journal of Geotechnical and Geoenvironmental Engineering, 128(11), pp. 907-916, 2002. 
[11] Li D, Selig ET. Method for Railroad Track Foundation Design. I: Development, Journal of Geotechnical and Geoenvironmental Engineering, 124(4), pp. 316-322, 1998.

[12] Li D, Selig ET, Cumulative Plastic Deformation for Fine-Grained Subgrade Soils. Geotechnical Engineering, 122(12), pp. 1006-1013, 1996. 